Conclusion Evaluation data and feedback demonstrated benefits to patients, referrers and increased awareness of services. Single point of referral enabled accurate data gathering and efficient use of service resources.

Demonstrated effective collaborative working. We learnt increased GP awareness across the locality may increase earlier referrals and clear feedback was required to referrers regarding priority of triage.

\section{P-205 WORKING AT SCALE... NOT TIPPING THE SCALES}

Bev Behmer, Duncan Fleming. Mountbatten, Isle of Wight, UK

\subsection{6/bmispcare-2019-HUKNC.227}

Background The Mountbatten Coordination Centre is central to our strategy moving forward. It is becoming the catalyst for working in different ways to future proof services, supporting people at scale with the services they need.

Aims Our coordination centre referral criteria of anyone with a life limiting disease or within their last five years of life, means we can reach more of our population earlier, preventing inappropriate admissions to the acute setting and enabling a planned approach to care. Our desire is to give the correct dose of care to each person at the right time, meaning our finite resources are used effectively and people aren't smothered in a blanket of care becoming 'a patient'.

Method The criterion for referral into our coordination centre is the last five years of life. All people referred are assessed and a plan put in place. The plan can range from light touch support, including access to our 24/7 support line, social programme and rapid response service, to the assignment of a specialist nurse. Our vision is that more people will fit in to the criteria of the former, enabling a planned approach, with patients' wishes achieved and stress and anxiety managed.

Results We have recently undertaken an evaluation of the Coordination Centre, this has resulted in some interesting findings. There is some evidence that it has had a positive impact on primary care with the amount of contacts decreasing after referral to the Coordination Centre. There have also been many pieces of anecdotal feedback from patients, family and professionals.

Conclusion We are using our limited resource to reach more people, when they need us; an increase of $50 \%$ in the past year. Helping them wherever, whenever and however is appropriate, to make the final years of their lives as rewarding as possible, giving all hope.

\section{P-206 CONTINUING HEALTHCARE FAST TRACK PROJECT - ONE YEAR ON}

Jane Baumeister, Gail Ewins, Lisa Morris. Garden House Hospice Care, Letchworth, UK

\subsection{6/bmjspcare-2019-HUKNC.228}

Background In April 2018, the hospice was commissioned to deliver care packages to patients who qualified for continuing healthcare (CHC) funding under fast track provision. Initially the project offered five virtual beds which enabled patients with a deteriorating and life limiting illness to remain at home by preventing avoidable admissions to hospital and facilitating early discharge from in-patient care, either hospital or hospice.

Aims of the service

- Ability to provide rapid discharge from acute sector;

- To deliver a comprehensive fast track CHC service for service users with palliative and end of life care needs based on their clinical need rather than diagnosis;

- To support people to live and die in their preferred place of care especially where this is identified as their own home;

- To prevent avoidable admission to hospital.

Method Commenced provision of five virtual beds in April 2018 and the level of care was based upon clinical need rather than being time specific. Care was provided by Health Care Assistants (HCAs) with the necessary skills to support this vulnerable group of people with specialist assessment being carried out by Registered Nurses. Care was offered up to a maximum of four times per day and was personalised and tailored to meet the needs of patients.

Results The service demonstrated:

- The ability to be responsive to patients' preferred place of care/death;

- The ability to support Community Teams;

- feedback from both patients and carers has been overwhelmingly positive;

- The smooth transition between hospice services including inpatient and day service.

Conclusion Following evaluation of the service and the positive feedback received from both staff, patients and relatives, the CCG have commissioned a further five virtual beds.

As of the 1 April 2019 the service can now provide care for 10 patients with a maximum of four visits per day.

\section{P-207 FAST TRACK IMPROVEMENT PROJECT: SIX MONTH REPORT (SEPT 2018 - MARCH 2019)}

Jane Moreland. University Hospitals of Derby and Burton NHS Foundation Trust, Derby, UK

\subsection{6/bmjspcare-2019-HUKNC.229}

Introduction Fast Track Continuing Healthcare (CHC) is a care package, funded and arranged by the NHS when a person is identified as having a 'rapidly deteriorating condition that may be entering a terminal phase of life' (National Framework for NHS Continuing Healthcare and NHS-funded Nursing Care, 2018).

Methodology Various initiatives were developed and implemented to provide guidance and improve the process:

- Direct electronic referral to CHC;

- Fast Track Operational guidelines detailing roles and responsibilities of staff;

- Integrated Discharge Team accountable for Fast Track referrals and discharge;

- Patient information leaflet;

- End of Life care: a guide - a booklet for people in the final stages of life and their carers (Macmillan Cancer Support and Mare Curie, 2015);

- Community services leaflet with contact telephone numbers;

- Discharge toolkit on the hospital intranet;

- Discharge prompt stickers for medical notes;

- End of life care facilitator attendance at daily ward board rounds; 
- Robust data collection and reporting on Fast Track discharges.

Implementation The audit data for this time period revealed a reduction in length of stay (LOS) from baseline data of six days (from 22 days to 16 days) and a reduction in admission to clinical decision to Fast Track by two days (from 15 days to 13 days) and the Fast Track process time has reduced from 7 days to 2.5 days.

Cost savings This equates to a saving of $£ 615,600$ based on the average cost of an acute bed price of $£ 300$ per day and 2052 beds saved (11 beds).

Conclusion Improvements have been made in the consistency and quality of data collection which is informing performance and evaluation of the process. Initial audit has shown that changes to how we approach Fast Track discharges have shown good progress in reduction in average length of stay and clinical decision making.

\section{P-208 A COLLABORATIVE PROJECT TO PROVIDE A RAPID PERSONALISED CARE SERVICE (RPCS) FOR PATIENTS}

${ }^{1}$ Judith Moore, ${ }^{2}$ Jackie Tritton, ${ }^{3}$ Fay Richardson. ${ }^{1}$ Rennie Grove Hospice Care, St Albans, UK; ${ }^{2}$ Peace Hospice Care, Watford, UK; ${ }^{3}$ Hospice of St Francis, Berkhamsted, UK

\subsection{6/bmjspcare-2019-HUKNC.230}

Background February 2018, the CCG reported an overspend of the CHC (Continuing Health Care) budget, with delays in funding approval and sourcing care packages, resulting in patients dying without a care package or with inappropriate hospital admissions.

Following the success of the West Hertfordshire Palliative Care Referral Centre (PCRC), (Hospice UK 2017 innovation in care award - a single point of access; three hospices and NHS community trust) three hospices collaboratively submitted a business case, proposing that any cost savings of 5\% and above would be reimbursed to the hospices. July 2018 the CCG commissioned (18-month pilot) delivering NHS CHC fast track pathway. The RPCS launched Sept 2018.

Aims of the RPCS Provide personalised care by trained end of life care carers.

- Based with the PCRC, so supporting the wider triage and coordination of Palliative/end of life care across West Herts;

- Receive hospital and community fast track referrals for community care packages

- Service provision from 8-8, 24/7 plus dedicated budget for overnight care, (sub-contracted with Marie Curie);

- More patients achieving their preferred place of care/death;

- No acute hospital discharge delays whilst waiting fast track care;

- Reduction of inappropriate hospital admission;

- Improved patient experience in last weeks of life;

- Opportunity to reach more people by the hospices.

Results to date (nine months)

- Deaths at home as a \% of all deaths has risen;

- Preferred place of death risen from $79 \%$ to $90 \%$;

- 99\% of care delivered within 48 hours;

- Only $2 \%$ of patients have exceeded the CHC fast track criteria of 12 weeks;

- $100 \%$ of care has been sourced by the hospices;

- Rise in the initiation of care packages and referrals from the previous year's data;
- Ability to change care provision at short notice ensuring patient-centred and cost effective;

- $95 \%$ of referrals met $\mathrm{CHC}$ criteria, demonstrating effective budget control.

Conclusions Based on the current figures and activity the pilot is delivering care for an increased number of patients for less care hours than previously seen in $\mathrm{CHC}$.

\section{P-209 DEVELOPMENT OF A COMMISSIONED FAST TRACK CONTINUING HEALTH CARE (CHC) SERVICE DELIVERED BY A HOSPICE}

Josephine Dedes, Ursula Reeve. St Luke's Hospice (Harrow and Brent), Harrow, UK

\subsection{6/bmjspcare-2019-HUKNC.231}

Background A review has identified the strength of the existing Hospice at Home service but weakness of contractual arrangements. The service has evolved from providing bridging to one which provides the initiation of all fast track care packages. The hospice has thus invested time and finance to support delivery of statutory CHC services.

Aims Pilot a dedicated hospice-led fast track brokerage service retaining current responsiveness and quality but delivered with $100 \%$ cost recovery for the hospice.

The pilot will respond to the NHS Long Term Plan (NHS England, 2019) and local CHC Strategic Improvement Plan (2019) requirements especially around expenditure by maximising the benefits of co-locating end of life and palliative care services.

Method Twelve month pilot will explore the:

- timeliness, quality and equity of access of a hospice-led service (Marie Curie, 2017; Marie Curie, 2019);

- interface between this service and a traditional hospice at home service;

- benefits of co-location with other hospice services;

- impact upon locality expenditure.

The service will receive, triage and assess all referrals for fast track $\mathrm{CHC}$ and initiate care.

Out of hours referrals will be picked up by the Hospice in the Home Service which will bridge care for a maximum of 72 hours.

Results A set of key performance indicators including the following will be used to measure outcomes:

- Range of packages delivered;

- \% of referrals who have care started within 24 hours of being able to receive care;

- \% of referrals that require Hospice in the Home Service to bridge care;

- $\%$ of patients utilising Personal Health Budgets.

Our intention is to demonstrate that timeliness and quality is best delivered by a hospice-led service and that this can positively impact equity of access and expenditure.

Conclusion The pilot demonstrates collaboration, has potential to positively impact patient outcomes, responds to policy requirements and could inform future commissioning recommendations. Since commencement of the pilot, initial results have identified potentially some significant benefits to patients, providers and commissioners. Therefore the poster presentation will focus on perceived outcomes. 